\title{
COMENTARIO EDITORIAL: Factores de riesgo y beneficios económicos del mejoramiento de la salud
}

\author{
Risk factors and economic benefits of health improvements
}

Adolfo Rubinstein*

\begin{abstract}
Resumen
Este artículo describe los resultados de la Encuesta Nacional de Factores de riesgo llevada a cabo en Argentina en 2005, el concepto de factor de riesgo múltiple utilizando como ejemplo al tabaquismo; y la importancia de las inversiones efectivas en salud para el desarrollo humano y el crecimiento económico. Al final del mismo el autor reflexiona sobre las diferencias en los resultados sanitarios entre los países desarrollados y los subdesarrollados, resaltando la menor calidad institucional de las organizaciones de salud en estos últimos y sus dificultades para hacer disponibles las tecnologías actuales para todos los potenciales beneficiarios.
\end{abstract}

\section{Abstract}

This article describes the results of the 2005 Argentinian National Risk Factors Survey, the multiple risk factor concept using smoking status as example, and the importance of effective investments in health for human development and economic growth. The author also explores the differences in health outcomes among developed and developing countries, focusing on worse institutional quality of health organizations in developing countries and their challenges in offering available technologies to all potencial beneficiaries.

\begin{abstract}
Palabras clave: factores de riesgo, desarrollo humano, crecimiento económico, desarrollo, calidad institucional. Key words: risk factors, human development, economic growth, development, institutional quality.
\end{abstract}

Rubinstein A. Factores de riesgo y beneficios económicos del mejoramiento de la salud. Evid. actual. práct. ambul; 10(4): 98-100, jul-agos.2007.

\section{Los factores de riesgo}

Globalmente, $45 \%$ de la mortalidad y $36 \%$ de la carga de enfermedad son atribuibles a 19 factores de riesgo (FR). Además, un número reducido de factores de riesgo (FR) contribuye de manera sustancial a la morbi-mortalidad de las enfermedades no transmisibles (ENT). Se estima que el $75 \%$ de las enfermedades cardiovasculares se deben a una dieta inadecuada, inactividad física y tabaquismo.

Un reciente informe de la Organización Mundial de la Salud evaluó 26 factores de riesgo seleccionados por su relevancia. Argentina pertenece a la zona de América con mortalidad intermedia (B) donde los principales factores de riesgo registrados para mortalidad fueron la presión arterial elevada, la obesidad, el abuso del alcohol y el tabaquismo.

La mayoría de los FR impacta en múltiples enfermedades y esto genera una oportunidad de controlar diferentes enfermedades o condiciones a través del control de un FR.

El tabaco es un claro ejemplo de factor de riesgo múltiple, ya que incide en una diversidad de condiciones como la enfermedad cardiovascular, el cáncer, la enfermedad pulmonar obstructiva crónica, el parto prematuro, etc. En Argentina, el tabaco -consumido por una tercera parte de la población mayor de 18 años- causa más de 40.000 muertes al año y explica $16 \%$ de las defunciones totales ${ }^{2}$. Además, se estima que en Argentina, el dinero gastado en el consumo de tabaco alcanzaría a pagar los gastos sanitarios de más de cinco millones de personas y que el costo de enfermedad atribuible podría significar casi $0,2 \%$ del PBI Argentino del año $2000^{3}$.

Además, como mencionamos previamente, un grupo reducido de FR explica la mayor parte de la morbimortalidad, brindando una oportunidad de intervención en la prevención y el control de estas condiciones. Se han realizado diversos estudios, en los que se demostró que la disminución de los factores de riesgo impactó directamente sobre la mortalidad de la población. Por ejemplo, en Karelia del Norte (Finlandia) las muertes cardiovasculares se redujeron hasta en un $50 \%$ y el $75 \%$ de esta disminución se atribuyó a las intervenciones sobre tres factores de riesgo (tabaquismo, hipertensión e hipercolesterolemia). Se estima que la reducción de $2 \mathrm{mmHg}$ en la presión arterial disminuye anualmente las muertes cardiovasculares en un $6 \%$.

\section{Encuesta Nacional de factores de riesgo}

A partir del año 2003, comenzó a implementarse por iniciativa del Ministerio de Salud de la Nación (MSN) la Encuesta Nacional de Factores de Riesgo, que valida un instrumento desarrollado por la Organización Panamericana de la Salud (OPS) y la Organización Mundial de la Salud (OMS) y cuya prueba piloto se llevó a cabo en Tierra del Fuego.

Durante 2005, el MSN y el Instituto Nacional de Estadística y Censos (INDEC) realizaron la encuesta a nivel nacional relevando 50.000 viviendas en todos los distritos del país. Se incluyó población de más de 18 años de ciudades de más de 5.000 habitantes.

Sus primeros resultados han sido recientemente publicados para el total del país y los diferentes distritos. Ver tabla 1.

\section{Los beneficios económicos del mejoramiento de la Salud}

Cuando el desempeño económico de los países se juzga sólo a través del Producto bruto interno (PBI) por habitante, es muy difícil diferenciar situaciones que difieren en sus condiciones de salud. Por ejemplo, un país cuyos ciudadanos gozan de vidas largas y saludables claramente tiene mejor desempeño social que un país con el mismo $\mathrm{PBI}$ pero cuyos ciudadanos viven menos y mas enfermos.

Existe una aceptación cada vez mayor por parte de la comunidad internacional de la importancia vital de las inversiones efectivas en salud para el desarrollo humano y el crecimiento económico.

El mejoramiento de la salud está siendo objeto de mayor atención dentro de las estrategias de lucha contra la pobreza, y los abordajes sectoriales se concentran cada vez más en los resultados sanitarios.

Se han creado nuevos mecanismos para la financiación de la salud (tales como la Alianza Mundial para Vacunas e Inmunización y el Fondo Mundial de Lucha contra el SIDA, la Tuberculosis y la Malaria) aprovechando nuevas alianzas en este ámbito, como la alianza "Alto a la Tuberculosis" y la alianza "Hacer Retroceder la Malaria". 
Cuadro 1: principales indicadores relevados en Argentina en la Encuesta Nacional de factores de riesgo.

\begin{tabular}{|c|c|c|}
\hline \multicolumn{2}{|l|}{ Indicador } & Prevalencia* \\
\hline \multicolumn{2}{|c|}{ Cobertura de salud solamente pública } & $34,9 \%$ \\
\hline \multicolumn{2}{|l|}{ Salud general regular o mala } & $19,9 \%$ \\
\hline \multicolumn{2}{|l|}{ Problemas de movilidad } & $10,9 \%$ \\
\hline \multicolumn{2}{|l|}{ Ansiedad y/o depresión } & $22,8 \%$ \\
\hline \multirow{9}{*}{$\begin{array}{l}\text { Principalmente asociados al } \\
\text { estilo de vida }\end{array}$} & Sedentarismo & $46,2 \%$ \\
\hline & Consumo de tabaco (18 a 64 años) & $33,4 \%$ \\
\hline & Sobrepeso y obesidad & $49,1 \%$ \\
\hline & Obesidad & $14,6 \%$ \\
\hline & Bajo consumo de frutas y verduras & $35,3 \%$ \\
\hline & Agregado de sal a las comidas & $45,2 \%$ \\
\hline & Consumo regular de riesgo de alcohol & $9,6 \%$ \\
\hline & Consumo episódico excesivo de alcohol & $10,1 \%$ \\
\hline & No haber usado alguna vez metodos anticonceptivos 0 de barrera en personas sin pareja estable & $27,2 \%$ \\
\hline \multirow{15}{*}{$\begin{array}{l}\text { Prácticas preventivas de } \\
\text { rastreo de factores de riesgo }\end{array}$} & Hipertensión arterial & \\
\hline & Control de la presión arterial el último año & $68,4 \%$ \\
\hline & Presión arterial elevada en las personas que refieren habérsela medido & $34,4 \%$ \\
\hline & Disli pidemia & \\
\hline & Control de colesterol (mayores de 35 años) & $70,7 \%$ \\
\hline & Colesterol elevado en personas que se controlaron & $27,8 \%$ \\
\hline & Trastornos de la glucemia & \\
\hline & Control de la glucemia & $69,3 \%$ \\
\hline & Diabetes o glucemia elevada en personas que refieren habérsela medido & $11,9 \%$ \\
\hline & Realización de Papanicolau & \\
\hline & Alguna vez & $74,4 \%$ \\
\hline & En los últimos dos años & $51,6 \%$ \\
\hline & Realización de mamografía luego de los 40 años & \\
\hline & Alguna vez & $62,2 \%$ \\
\hline & En los últimos dos años & $42,5 \%$ \\
\hline \multirow{5}{*}{$\begin{array}{l}\text { Relacionados con lesiones } \\
\text { intencionales y no intencionales } \\
\text { y con secuelas post-traumáticas }\end{array}$} & No haber usado alguna vez el cinturón de seguridad & $48,0 \%$ \\
\hline & Presencia de armas en el entorno & $9,8 \%$ \\
\hline & Haber sido víctima de robo a mano armada últimos 12 meses & $5,6 \%$ \\
\hline & Haber sido testigo de robo a mano armada & $9,0 \%$ \\
\hline & Haber sido golpeado en los últimos 12 meses & $3,5 \%$ \\
\hline
\end{tabular}

*Definición de prevalencia: proporción de casos en la población total en un punto determinado en el tiempo. Fuente: Encuesta Nacional de Factores de Riesgo. Ministerio de Salud de la Nación. 2005.

La tarea desarrollada en el ámbito nacional e internacional para promover el mejoramiento de la salud de los pobres se plasma mejor que de ninguna otra forma en el amplio respaldo a los Objetivos de Desarrollo del Milenio (ODM) de las Naciones Unidas. Recientemente, la comisión sobre Macroeconomía y Salud de las Naciones Unidas en su informe de 2001, mostró clara evidencia empírica de la relación directa entre salud y producción, y desarrollo económico, analizando entre otros ejemplos, el impacto del aumento de la incidencia de paludismo y VIH/SIDA sobre el desarrollo económico de países del África Subsahariana.

En este informe y en otros ulteriores, se demuestra que la inversión en Salud es crítica para el desarrollo económico de los países pobres debido a su impacto sobre la productividad laboral, la capacidad de aprendizaje en la escuela y el crecimiento físico, emocional e intelectual; es decir mayor crecimiento de lo que se conoce como "capital humano".

El focalizar las intervenciones en los países subdesarrollados sobre aquellas condiciones de alta prevalencia e impacto es altamente redituable, ya que pocas condiciones son responsables de la mayoría de la carga de enfermedad (VIH/SIDA, paludismo, tuberculosis, enfermedades infecciosas en la infancia, condiciones maternas y perinatales, enfermedades rela- cionados con el tabaco y déficit de micronutrientes).

La inversión en Salud Reproductiva, incluyendo planificación familiar y acceso a anticonceptivos por ejemplo, ha demostrado ser una de las estrategias más costo-efectivas para acompañar las intervenciones para el control de las enfermedades. Esta combinación de intervenciones ha demostrado reducir la tasa de fecundidad, aumentar la inversión en salud y educación por niño y reducir el crecimiento de la población.

Lamentablemente, la inversión actual en los países pobres es insuficiente para financiar incluso los servicios esenciales de salud pública, que al menos necesitan de 30 a 40 dólares por año .

\section{El saber cómo y el saber como hacer}

La comprensión de las razones que explican la reducción de la mortalidad producida en el siglo XX es fundamental para diseñar políticas de salud que permitan trabajar sobre las poblaciones de muchos países en los cuales esos resultados están lejos de ser alcanzados, y que representan entre el 25 y el $30 \%$ de la población mundial. Si bien el aumento del ingreso económico de las familias que comenzó a darse en las últimas 
décadas del siglo XIX, tuvo probablemente un impacto neto positivo en buena medida por el mejoramiento de la nutrición y las condiciones sanitarias ambientales, parte de este beneficio fue contrarrestado por el incremento de las enfermedades infecciosas, producto de la mayor urbanización ocurrida en sus últimas décadas y las primeras del siglo XX. La reducción de la mortalidad en el siglo pasado, mucho más acelerada que en el precedente, guarda clara relación con el progreso técnico, aunque es importante también señalar que los productos obtenidos a través del avance científico y tecnológico necesitan a su vez de profundos cambios institucionales en los servicios de salud para que se traduzcan en mejoramientos de la cantidad y calidad de vida.
En este sentido, muchas de las diferencias en los resultados sanitarios que pueden mostrar los países desarrollados en relación a los subdesarrollados no se deben sólo a las diferencias tecnológicas sino fundamentalmente a la menor calidad institucional de las organizaciones de salud en estos últimos. No sólo se trata de generar o adquirir el conocimiento tecnológico o la tecnología en si, sino también de difundirlo apropiadamente para que se ponga en práctica y se implemente para todos los potenciales beneficiarios. El saber cómo hacer ("know-do") y no sólo el saber cómo ("know-how") también explican parte de la brecha tecnológica que nos separa cada vez más de los países centrales ("know-do gap").

Recibido el 10/06/07 y aceptado el 16/07/07

\section{Referencias}

1.The world health report 2002. Reducing Risks, Promoting Healthy Life. WHO. http://www.who.int/whr/2002/en/

2. Mortalidad anual atribuible al tabaquismo. Pitarque R. Publicación del Ministerio de Salud de la Nación. 2000.

3. Conte Grand M y col. Costo económico de la mortalidad atribuible al tabaquismo. Programa Vigi+A. Ministerio de Salud de la Nación. 2000

4. Comisión de Macroeconomía y Salud. WHO 2001. http://www.who.int/macrohealth/

\section{INFO - EVIDENCIA}

\section{¿Qué es Evidencia, Actualización en la Práctica Ambulatoria?}

Una publicación independiente editada cada dos meses desde 1997 por la Fundación MF, organización sin fines de lucro dedicada a promover el desarrollo de la medicina familiar y la atención primaria de la salud.

¿Cuál es el objetivo de Evidencia?

Contribuir a la educación continua y a la actualización de los profesionales de la salud de la región en el área de la atención ambulatoria.

\section{Contenidos de Evidencia}

- Información independiente, actualizada y resumida en forma sencilla y en castellano sobre temas relevantes de la atención ambulatoria.

- Artículos seleccionados por su calidad y relevancia clínica, resumidos y comentados críticamente por destacados profesionales del área.

- Revisiones sobre temas clínicos, epidemiológicos, sanitarios o humanísticos, de importancia para la práctica ambulatoria.

- Notas farmacológicas para la actualización rápida de temas de terapéutica clínica.

- Discusión de casos clínicos con herramientas de Medicina Basada en la Evidencia.

- Glosario de términos de epidemiología y medicina basada en la evidencia

¿Cómo es posible acceder a Evidencia?

Por suscripción a la edición en papel, o bien por Internet, en nuestro sitio: http://www.evidencia.org 